\title{
Harmonic Analysis of Local Operators
}

Detlev Buchholz

II. Institut für Theoretische Physik, Universität Hamburg, Luruper Chaussee 149, D-2000 Hamburg 50, Federal Republic of Germany

\begin{abstract}
The spatial Fourier transforms of local operators are analysed. It is shown that the Fourier components for non-zero momentum form weakly square integrable functions in all states of finite energy. Moreover, there hold uniform bounds for the respective $L^{2}$-norms. The relevance of this result is illustrated in collision theory.
\end{abstract}

\section{Introduction}

The interplay between locality and the spectrum condition is one of the basic ingredients in many investigations of quantum field theory. The link between these structures is provided by harmonic (Fourier) analysis. It is the aim of the present paper to exhibit regularity properties of the spatial Fourier transforms of local operators which have escaped observation so far. These regularity conditions greatly simplify the analysis, notably in collision theory.

The setting and the notation used in this paper are standard. Let $\mathscr{H}$ be a Hilbert space, let $\mathfrak{A} \subset \mathscr{B}(\mathscr{H})$ be a ${ }^{*}$-algebra of local operators, and let $U$ be a continuous unitary representation of the space-time translations $x \in \mathbb{R}^{s+1}$ which acts on $\mathscr{H}$ and satisfies the relativistic spectrum condition [1]. We recall that the condition of locality implies that for each operator $A \in \mathfrak{A}$ there exists some finite distance $d>0$ such that

$$
\left[A(x), A^{*}\right]=0 \quad \text { if } \quad|\mathbf{x}| \geq\left|x_{0}\right|+d .
$$

Here $\mathbf{x}, x_{0}$ denote the space and time part of the translation $x$ with respect to a fixed coordinate system and we have introduced the notation

$$
B(x)=U(x) B U(x)^{-1} \quad \text { for } \quad B \in \mathscr{B}(\mathscr{H}) .
$$

We write $B(\mathbf{x})$ if the time component $x_{0}$ of $x$ in relation (2) is zero, and similarly $B\left(x_{0}\right)$ if $\mathbf{x}=0$. 
Within this general setting we want to study the properties of the Fourier transforms

$$
\tilde{A}(\mathbf{p})=(2 \pi)^{-s / 2} \int d^{s} x e^{i \mathbf{p x}} A(\mathbf{x}), \quad A \in \mathfrak{A},
$$

which are defined in the sense of operator valued distributions. We mention as an aside that the support of $\tilde{A}(\mathbf{p})$ coincides with $\mathbb{R}^{s}$ if the local operator $A \in \mathfrak{A}$ is not an element of the center of $\mathfrak{A}$. This is an immediate consequence of the locality condition (1) and the Paley-Wiener theorem.

We will see that the Fourier transforms $\tilde{A}(\mathbf{p})$ enjoy certain specific regularity properties in states of finite energy. Let $P(E), E>0$ be the spectral projection of the generator $H$ of the time translations (the Hamiltonian) corresponding to the spectrum in the interval $[0, E]$ and let $\omega$ be any normal state on $\mathscr{B}(\mathscr{H})$ whose support projection is contained in $P(E)$. It will turn out that for any $\delta>0$ the restriction of $\omega(\tilde{A}(\mathbf{p}))$ to the domain $\{\mathbf{p}:|\mathbf{p}| \geq \delta\}$ is represented by some square integrable function. Moreover, the $L^{2}$-norm of this function is, for fixed $E$ and $\delta$, uniformly bounded in $\omega$. We have also control on the nature of the singularities of these functions at $\mathbf{p}=0$. They exhibit an at most power like singularity whose strength depends on the dimension $s$ of space. These facts will be established in Sect. 2.

We emphasize that we depend in our analysis neither on the existence of a vacuum state nor on the existence of Lorentz transformations. In fact, our analysis can be extended to arbitrary dynamical systems, provided the spectrum of $U$ lies in some positive cone and the locality condition (1) is replaced by some sufficiently strong form of asymptotic abelianess. The precise requirements will become clear in context.

Besides adding information to the general harmonic analysis of automorphism groups [2] our results are useful in applications. In Sect. 3 we will indicate how the a priori information about the Fourier transforms $\tilde{A}(\mathbf{p})$ simplifies the analysis of asymptotic fields and observables in collision theory. In a forthcoming paper [3] the present methods will be applied in an investigation of the asymptotic vacuum structure in quantum field theory.

\section{Analysis}

The essential ingredient in our analysis is the following lemma based on "positivity of the metric." Similar ideas have been used in [4], but the present result seems to be new.

Lemma 2.1. Let $B \in \mathscr{B}(\mathscr{H})$, let $n \in \mathbb{N}$, and let $P_{n}$ be the orthogonal projection onto the kernel of $B^{n}$. Then

$$
\left\|P_{n} B^{*} B P_{n}\right\| \leq(n-1) \cdot\left\|\left[B, B^{*}\right]\right\| .
$$

Proof. The proof is given by induction in $n$. For $n=1$ the statement is trivial. The step from $(n-1)$ to $n$ is accomplished as follows: let $\omega$ be any state on 
$\mathscr{B}(\mathscr{H})$ which is induced by some vector in the subspace $P_{n} \mathscr{H}$. (We assume that $P_{n} \neq 0$, otherwise the statement is trivial.) Setting $Q=B^{*} B$ we have

$$
\begin{aligned}
\omega(Q \cdot Q) & =\omega\left(B^{*}\left[B, B^{*}\right] B\right)+\omega\left(B^{*} Q B\right) \\
& \leq \omega(Q) \cdot\left\|\left[B, B^{*}\right]\right\|+\omega\left(B^{*} Q B\right) \\
& =\omega(Q) \cdot\left\|\left[B, B^{*}\right]\right\|+\omega\left(B^{*} P_{n-1} Q P_{n-1} B\right),
\end{aligned}
$$

where in the last step we made use of the fact that the positive functional $\omega\left(B^{*} . B\right)$ is induced by some vector in $B P_{n} \mathscr{H} \subseteq P_{n-1} \mathscr{H}$. It thus follows from the induction hypothesis that

$$
\omega(Q \cdot Q) \leq(n-1) \cdot\left\|\left[B, B^{*}\right]\right\| \cdot \omega(Q) .
$$

On the other hand we have $\omega(Q \cdot Q) \geq \omega(Q)^{2}$ since $\omega$ is a state, and consequently

$$
\omega(Q) \leq(n-1) \cdot\left\|\left[B, B^{*}\right]\right\| .
$$

The statement now follows by taking the supremum over all states $\omega$ which are induced by vectors in $P_{n} \mathscr{H}$.

There exist several variants of this lemma. For later use we mention

Lemma 2.2. Let $B \in \mathscr{B}(\mathscr{H})$, let $n \in \mathbb{N}$, and let $P_{n}$ be the orthogonal projection onto the intersection of the kernels of the $n$-fold products $B\left(\mathbf{x}_{1}\right) \ldots B\left(\mathbf{x}_{n}\right)$ for arbitrary $\mathbf{x}_{1}, \ldots, \mathbf{x}_{n} \in \mathbb{R}^{s}$. There then holds for each compact subset $\mathbf{K} \subset \mathbb{R}^{s}$ the estimate

$$
\left\|P_{n} \int_{\mathbf{K}} d^{s} x\left(B^{*} B\right)(\mathbf{x}) P_{n}\right\| \leq(n-1) \cdot \sup \int_{\Delta \mathbf{K}} d^{s} x\left\|\left[B(\mathbf{x}), B^{*}\right] \Psi\right\|,
$$

where $\Delta \mathbf{K}=\{\mathbf{x}-\mathbf{y}: \mathbf{x}, \mathbf{y} \in \mathbf{K}\}$, and the supremum is to be taken with respect to all unit vectors $\Psi \in P_{n-1} \mathscr{H}$.

The proof of this statement is given in the Appendix. The result is of interest if the norm of the commutator $\left[B(\mathbf{x}), B^{*}\right]$ decreases sufficiently rapidly for large $\mathbf{x}$. One can then replace the region $\Delta \mathbf{K}$ in (4) by $\mathbb{R}^{s}$ and arrives at bounds for the integral appearing on the left-hand side of this relation which are uniform in $\mathbf{K}$.

In the next step we construct from any given local operator $A \in \mathfrak{A}$ certain specific operators to which we can apply Lemma 2.1. Let $\delta>0$ and let $\mathbf{e} \in \mathbb{R}^{s}$ be some unit vector. Picking any $f \in \mathscr{S}(\mathbb{R})$ whose Fourier transform $\tilde{f}\left(p_{0}\right)$ has support in $\left\{p_{0}:-\infty<p_{0} \leq \delta / 2\right\}$ and any $g \in \mathscr{S}\left(\mathbb{R}^{s}\right)$ whose Fourier transform has support in $\{\mathbf{p}: \mathbf{p} \cdot \mathbf{e} \geq \delta\}$, we define

$$
A_{\delta}=\int d x f\left(x_{0}\right) g(\mathbf{x}) A(x) .
$$

In the subsequent lemma we establish properties of these operators which are a consequence of the spectrum condition and locality.

Lemma 2.3. Let $A_{\delta}$ be the operator defined in relation (5). Then:

$$
\left(A_{\delta}\right)^{n} P(E)=0
$$


if $n \in \mathbb{N}$ satisfies $n>4 E / \delta$. Here $P(E), E>0$ are the spectral projections of the Hamiltonian corresponding to the spectrum in $[0, E]$.

ii)

$$
\left\|\left[A_{\delta}^{*}, A_{\delta}\right]\right\| \leq 4^{s} \Omega_{s}\left\|f_{s}\right\|_{1}\|f\|_{1}\|g\|_{2}^{2} \cdot\|A\|^{2} .
$$

Here $\Omega_{s}$ denotes the volume of the unit ball in $\mathbb{R}^{s},\|\cdot\|_{p}$ denotes the $L^{p}$-norm of the respective function, and $f_{s}$ is the function defined by $f_{s}\left(x_{0}\right)=\left(\left|x_{0}\right|^{s}+(d / 2)^{s}\right) \cdot f\left(x_{0}\right)$, where $d$ is the distance appearing in the locality condition (1) for the operator $A$.

Proof. i) Let $\Delta \subset \mathbb{R}^{s+1}$ be any Borel set. We denote by $\mathscr{H}(\Delta)$ the spectral subspace of $\mathscr{H}$ corresponding to the joint spectrum of the generators of $U$ in $\Delta$. Because of the relativistic spectrum condition we have $P(E) \mathscr{H}=\mathscr{H}(\{p: 0 \leq$ $\left.\left.|\mathbf{p}| \leq p_{0} \leq E\right\}\right)$. On the other hand, the space-time Fourier transform of $A_{\delta}$ has by construction support in $\left\{k: k_{0} \leq \delta / 2, \mathbf{k} \cdot \mathbf{e} \geq \delta\right\}$. Hence $\left(A_{\delta}\right)^{n} P(E) \mathscr{H} \subseteq \mathscr{H}\left(\Delta_{n}\right)$, where

$$
\Delta_{n}=\left\{p+k: 0 \leq|\mathbf{p}| \leq p_{0} \leq E, k_{0} \leq n \delta / 2, \mathbf{k} \cdot \mathbf{e} \geq n \delta\right\} .
$$

The condition on $n$ in the lemma implies that $\Delta_{n}$ is disjoint from the closed forward lightcone, and applying the relativistic spectrum condition a second time we conclude that $\mathscr{H}\left(\Delta_{n}\right)=\{0\}$.

ii) Let $\Phi, \Psi \in \mathscr{H}$ be arbitrary unit vectors. It follows from the definition of the operators $A_{\delta}$ and the locality condition (1) that

$$
\begin{aligned}
& \left|\left(\Phi,\left[A_{\delta}^{*}, A_{\delta}\right] \Psi\right)\right| \\
& \quad \leq \int d x \int d y\left|f\left(x_{0}\right)\right|\left|f\left(y_{0}\right)\right| \cdot 2^{-1}\left(|g(\mathbf{x})|^{2}+|g(\mathbf{y})|^{2}\right) \cdot\left|\left(\Phi,\left[A^{*}(x), A(y)\right] \Psi\right)\right| \\
& \quad \leq 2 \Omega_{s} \cdot\|g\|_{2}^{2}\|A\|^{2} \cdot \int d x_{0} \int d y_{0}\left|f\left(x_{0}\right)\right|\left|f\left(y_{0}\right)\right|\left(\left|x_{0}-y_{0}\right|^{2}+d^{2}\right)^{s / 2} .
\end{aligned}
$$

Making use of the fact that

$$
\left(\left|x_{0}-y_{0}\right|^{2}+d^{2}\right)^{2 / s} \leq 4^{2-1}\left(\left|x_{0}\right|^{s}+\left|y_{0}\right|^{s}+2(d / 2)^{s}\right)
$$

the bound given in the lemma follows.

Now let $\delta>0$, let $\mathbf{e} \in \mathbb{R}^{s}$ be some unit vector, let $g \in \mathscr{S}\left(\mathbb{R}^{s}\right)$ be any test function whose Fourier transform has support in $\{\mathbf{p}: \mathbf{p} \cdot \mathbf{e} \geq \delta\}$, and let $A \in \mathfrak{A}$ be some local operator. We will study the continuity properties of the map $g \rightarrow P(E) A(g) P(E)$, where $A(g)=\int d^{s} x g(\mathbf{x}) A(\mathbf{x})$. To this end we choose test functions $f^{ \pm} \in \mathscr{S}(\mathbb{R})$ whose Fourier transforms $\tilde{f}^{ \pm}\left(p_{0}\right)$ vanish for $\mp p_{0} \geq \delta / 2$ and add up to 1 for $\left|p_{0}\right| \leq E$. A for our purposes convenient choice is

$$
f^{ \pm}\left(x_{0}\right)= \pm\left(i x_{0}\right)^{-1} \cdot\left(h\left(\delta x_{0} / 2\right)-e^{\mp 2 i E x_{0}} h\left(E x_{0}\right)\right),
$$

where $h \in \mathscr{S}(\mathbb{R})$ is a fixed real testfunction such that $h(0)=1$ and the Fourier transform of $h$ has support in $[-1,1]$. We will make use of the facts that $f^{+^{*}}=f^{-}$and that

$$
\begin{gathered}
\int d x_{0}\left|x_{0}\right|^{s}\left|f^{-}\left(x_{0}\right)\right| \leq\left((2 / \delta)^{s}+(1 / E)^{s}\right) \cdot \int d x_{0}\left|x_{0}\right|^{s-1}\left|h\left(x_{0}\right)\right| \\
\int d x_{0}\left|f^{-}\left(x_{0}\right)\right| \leq 2 \cdot \int d x_{0}\left|h\left(x_{0}\right)\right|+|\ln (2 E / \delta)| \cdot \int d x_{0}\left|\frac{d}{d x_{0}} h\left(x_{0}\right)\right| .
\end{gathered}
$$


These bounds can be derived from (6) by a straightforward computation. One could proceed further and determine, for given dimension $s$, explicit numerical values for the integrals involving $h$ by choosing suitable functions, but this is not necessary here.

With the help of the test functions $f^{ \pm}$we construct the operators

$$
A_{\delta}^{ \pm}=\int d x f^{ \pm}\left(x_{0}\right) g(\mathbf{x}) A(x) .
$$

The operator $A_{\delta}^{-}$is of the type considered in Lemma 2.3. Since $f^{+^{*}}=f^{-}$and since the Fourier transform of $g(\mathbf{x})^{*}$ has support in $\{\mathbf{p}: \mathbf{p} \cdot(-\mathbf{e}) \geq \delta\}$, the same is true for $A_{\delta}^{+*}$. Moreover, since the Fourier transform of $\left(f^{+}+f^{-}\right)$is equal to 1 for $\left|p_{0}\right| \leq E$, we have

$$
P(E) A(g) P(E)=P(E)\left(A_{\delta}^{+}+A_{\delta}^{-}\right) P(E),
$$

and consequently

$$
\|P(E) A(g) P(E)\| \leq\left\|A_{\delta}^{+*} P(E)\right\|+\left\|A_{\delta}^{-} P(E)\right\| .
$$

From the first part of Lemma 2.3 we see that Lemma 2.1 can be applied to the expressions appearing on the right-hand side of relation (10), giving

$$
\begin{aligned}
\|P(E) A(g) P(E)\| & \leq(4 E / \delta)^{1 / 2}\left(\left\|\left[A_{\delta}^{+}, A_{\delta}^{+*}\right]\right\|^{1 / 2}+\left\|\left[A_{\delta}^{-*}, A_{\delta}^{-}\right]\right\|^{1 / 2}\right) \\
& \leq\left(4^{s+2} \Omega_{s}(E / \delta)\left\|f_{s}^{-}\right\|_{1}\left\|f^{-}\right\|_{1}\right)^{1 / 2}\|g\|_{2}\|A\| .
\end{aligned}
$$

In the latter inequality we used the second part of Lemma 2.3. Making use of the bounds on $f^{-}$given in (7) and of the facts that $\|P(E) A(g) P(E)\|=0$ if $\delta>2 E$ and $\ln x \leq \lambda^{-1} x^{\lambda}$ if $x>0, \lambda>0$ we obtain for any $0<\varepsilon \leq 1$,

$$
\|P(E) A(g) P(E)\| \leq C_{s}\left(\delta^{-s}+d^{s}\right)^{1 / 2} \cdot \varepsilon^{-1}(E / \delta)^{\varepsilon+1 / 2} \cdot\|g\|_{2}\|A\| .
$$

Notation. Here and in the subsequent analysis $C_{s}$ denotes some numerical constant which is independent of all quantities in the respective formulas, but which depends on the dimension $s$ of space.

Up to this point we made use of the assumption that the Fourier transform of $g$ has support in some half space $\{\mathbf{p}: \mathbf{p} \cdot \mathbf{e} \geq \delta\}$. Now let $g$ be any test function whose Fourier transform has support in $\{\mathbf{p}:|\mathbf{p}| \geq \delta\}$. We then find by a standard partition-of-unity argument test functions $g_{i}$ such that $\sum_{i} g_{i}=g$, $\left\|g_{i}\right\|_{2} \leq\|g\|_{2}$, and the Fourier transform of each $g_{i}$ has support in some half space $\left\{\mathbf{p}: \mathbf{p} \cdot \mathbf{e}_{i} \geq \delta / 2\right\}$. The maximal number of functions $g_{i}$ needed in this partition depends only on the dimension $s$ of space. From this we see that the bound (12) holds for arbitrary test functions $g$ whose Fourier transforms vanish in the ball $\{\mathbf{p}:|\mathbf{p}| \leq \delta\}$.

Finally, we decompose $g$ into a sum of test functions whose Fourier transforms have support in shrinking concentric shells about the origin and apply relation (12) to each individual term. For the resulting sum we then find by a straightforward application of the Cauchy-Schwarz inequality upper bounds leading to our main technical result: 
Proposition 2.4. Let $A$ be any local operator satisfying condition (1), let $P(E)$ be the spectral projections of the Hamiltonian corresponding to the spectrum in $[0, E]$, and let $g \in \mathscr{S}\left(\mathbb{R}^{s}\right)$ be any test function whose Fourier transform vanishes at the origin. Then

$$
\|P(E) A(g) P(E)\| \leq C_{s} \varepsilon^{-3 / 2}\left(\int d^{s} p(E /|\mathbf{p}|)^{1+\varepsilon}\left(|\mathbf{p}|^{-s}+d^{s}\right)|\tilde{g}(\mathbf{p})|^{2}\right)^{1 / 2}\|A\|
$$

for any $0<\varepsilon<1$.

The properties of the Fourier transforms of local operators mentioned in the introduction are a simple consequence of this result. This is shown in

Theorem 2.5. Let $\varphi$ be any normal linear functional on $\mathscr{B}(\mathscr{H})$ whose right and left support projection are contained in $P(E)$, let $A$ be any local operator, and let $\delta>0$. Then the restriction of the distribution $\varphi(\tilde{A}(\mathbf{p}))$ to the domain $\{\mathbf{p}:|\mathbf{p}| \geq \delta\}$ is represented by a square-integrable function. In fact, one has for any $0<\varepsilon<1$

$$
\int d^{s} p|\mathbf{p}|^{s+1+\varepsilon}|\varphi(\tilde{A}(\mathbf{p}))|^{2} \leq C_{s} \varepsilon^{-3} E^{1+\varepsilon}\left(1+(E d)^{s}\right)\|\varphi\|^{2}\|A\|^{2} .
$$

Proof. Let $L_{\varepsilon}^{2}, 0<\varepsilon<1$ be the Hilbert space of (classes of) functions $h$ on $\mathbb{R}^{s}$ for which

$$
\|h\|_{2, \varepsilon}^{2}=\int d^{s} p|\mathbf{p}|^{-s-1-\varepsilon}|h(\mathbf{p})|^{2}<\infty
$$

It follows from standard arguments that the subspace of test functions in $\mathscr{S}\left(\mathbb{R}^{s}\right)$ which vanish at $\mathbf{p}=0$ is dense in each $L_{\varepsilon}^{2}$. Since

$$
|\varphi(A(g))| \leq\|\varphi\| \cdot\|P(E) A(g) P(E)\|
$$

and since the distribution $\varphi(\tilde{A}(\mathbf{p}))$ has support in the ball $\{\mathbf{p}:|\mathbf{p}| \leq 2 E\}$ as a consequence of the spectrum condition we infer from the preceding proposition that

$$
|\varphi(A(g))|^{2} \leq C_{s} \varepsilon^{-3} E^{1+\varepsilon} \cdot\left(1+(E d)^{S}\right) \cdot\|\tilde{g}\|_{2, \varepsilon}^{2} \cdot\|\varphi\|^{2}\|A\|^{2} .
$$

The statement now follows from Riesz' theorem.

It is evident that there holds a similar statement for functionals $\varphi$ which are in the domain of sufficiently large powers of $H$ under simultaneous left and right multiplication. Moreover, the assumption that $A$ is local can be replaced by the requirement that $A$ is quasi-local of sufficiently large order [5, Sect.2]. One may even relax the condition that $A$ is bounded and replace it by the assumption that $A$ satisfies energy bounds of the form $\|A P(E)\|<\infty,\left\|A^{*} P(E)\right\| \mid<\infty$. Since these generalizations are straightforward we refrain from giving details.

So far we have treated $A(g)$ effectively as a bilinear form, and our estimates led us to uniform bounds on this form. If $A\left(x_{0}\right)$ is differentiable with respect to the time translations $x_{0}$ we can proceed further and give bounds on $A(g)$ in the operator sense. Denoting the derivative of $A\left(x_{0}\right)$ at $x_{0}=0$ by $\dot{A}$ we have 
Theorem 2.6. Let $A$ be any local operator such that $x_{0} \rightarrow A\left(x_{0}\right)$ is strongly differentiable, let $g \in \mathscr{S}\left(\mathbb{R}^{s}\right)$ be any test function whose Fourier transform vanishes at the origin, and let $\lambda>0$. Then

$$
\left\|A(g)(1+\lambda H)^{-1-\varepsilon}\right\| \leq C_{s}(\|A\|+\lambda\|\dot{A}\|) \varepsilon^{-2}\left(\int d^{s} p(\lambda|\mathbf{p}|)^{-1-\varepsilon / 2}\left(|\mathbf{p}|^{-s}+d^{s}\right)|\tilde{g}(\mathbf{p})|^{2}\right)^{1 / 2}
$$

for any $0<\varepsilon<1$, and the same bound holds for $\left\|(1+\lambda H)^{-1-\varepsilon} A(g)\right\|$.

Proof. Making use of the spectral theorem for $H$ it follows from Proposition 2.4 that

$$
\begin{aligned}
\left\|(1+\lambda H)^{-(1+\varepsilon) / 2} A(g)(1+\lambda H)^{-(1+\varepsilon) / 2}\right\| \\
\quad \leq C_{s}\|A\| \varepsilon^{-2}\left(\int d^{s} p(\lambda|\mathbf{p}|)^{-1-\varepsilon / 2}\left(|\mathbf{p}|^{-s}+d^{s}\right)|\tilde{g}(\mathbf{p})|^{2}\right)^{1 / 2},
\end{aligned}
$$

and the same inequality holds if $A$ is replaced by $\dot{A}$, because $\dot{A}$ is a local operator with the same localization properties as $A$. On the basis of these estimates and the fact that $[(1+\lambda H), A(g)]=i \lambda \dot{A}(g)$ we conclude that the norm of the function

$$
z \in \mathbb{C} \rightarrow(1+\lambda H)^{z} A(g)(1+\lambda H)^{-z-1-\varepsilon}
$$

is bounded from above by the expression given on the right-hand side of the inequality in the theorem if $\operatorname{Re} z=-(1+\varepsilon) / 2$ or $\operatorname{Re} z=(1-\varepsilon) / 2$, respectively. The first half of the statement now follows from the three line theorem, cp. [6]. The second half is a consequence of the fact that $\left\|(1+\lambda H)^{-1-\varepsilon} A(\mathrm{~g})\right\|=$ $\left\|A^{*}\left(g^{*}\right)(1+\lambda H)^{-1-\varepsilon}\right\|$ and that $A^{*}$ has the same differentiability and localization properties as $A$.

It is an immediate consequence of this theorem that $A(g)$ can be defined as a closable unbounded operator for all functions $g$ for which the $L^{2}$-norm given in the theorem is finite. Moreover, there hold energy bounds for $A(g)$ which are almost linear in $H$. Again, there exist analogous results for quasi-local, respectively unbounded operators $A$.

As can be seen in free field theory, our general results on the properties of the Fourier transforms $\tilde{A}(\mathbf{p})$ are about optimal for non-zero momentum $\mathbf{p}$. Yet our bounds on the behaviour of $\tilde{A}(\mathbf{p})$ at $\mathbf{p}=0$ seem to be too conservative. The origin for these weaker results is our crude estimate of the integrals $\int d^{s} x\left|\left(\Phi,\left[A^{*}\left(x_{0}, \mathbf{x}\right), A\right] \Psi\right)\right|$ in Lemma 2.3 . Making only use of locality, we gave an upper bound for these integrals which increases like $\left|x_{0}\right|^{s}$ for large $\left|x_{0}\right|$. If this bound would be saturated it would mean that sup ess $\left\{\left|\left(\Phi,\left[A^{*}\left(x_{0}, \mathbf{x}\right), A\right] \Psi\right)\right|: \mathbf{x} \in\right.$ $\mathbb{R}^{s}$ \} does not tend to 0 at asymptotic times $x_{0}$. On the other hand one expects that this expression decreases (disregarding theories of massless particles in two space-time dimensions) like an inverse power of $\left|x_{0}\right|$ because of dispersive effects. With such an input one can establish stronger results which are relevant for a discussion of the asymptotic vacuum structure of states in quantum field theory [3]. 


\section{Applications}

In order to illustrate the utility of our general results for the structural analysis in quantum field theory we briefly reconsider here the construction of asymptotic fields and observables in collision theory.

According to the basic ideas of Lehmann, Symanzik and Zimmermann [7] one approaches the construction of asymptotic fields for a given particle type by averaging suitable local (Bose or Fermi) operators $A$ with solutions of the KleinGordon equation, respectively of the wave equation. The resulting operators are of the form

$$
A_{t}(g)=\left.\int d^{s} x g\left(x_{0}, \mathbf{x}\right) A\left(x_{0}, \mathbf{x}\right)\right|_{x_{0}=t},
$$

where

$$
g\left(x_{0}, \mathbf{x}\right)=(2 \pi)^{-s / 2} \int d^{s} p \tilde{g}(\mathbf{p}) e^{-i E x_{0}+i \mathbf{p x}} .
$$

Here $E=\left(|\mathbf{p}|^{2}+m^{2}\right)^{1 / 2}$ and $m \geq 0$ is the mass of the respective particle. One then argues that the operators $A_{t}(\mathrm{~g})$ converge weakly in the limit of asymptotic times $t$ to the desired asymptotic fields on some dense set of vectors. By taking suitable time averages of $A_{t}(\mathrm{~g})$ one can also achieve strong convergence.

In the case of Bosons the actual justification of this method is plagued by domain problems due to the fact that the asymptotic fields are unbounded operators. In the existing arguments these difficulties are handled in a fairly involved manner, cp. [4, Sect. 15] and [8]. Here the present results lead to considerable simplifications.

It follows from Theorem 2.6 that for any $0<\varepsilon<1$ the norms $\| A_{t}(g)(1+$ $\lambda H)^{-1-\varepsilon} \|$ and $\left\|A_{t}(g)^{*}(1+\lambda H)^{-1-\varepsilon}\right\|$ stay bounded in the limit of asymptotic times $t$, provided the operator $A$ and the initial wave function $\left.g\left(x_{0}, \mathbf{x}\right)\right|_{x_{0}=0}$ comply with the premises of the theorem. Hence the sequence of operators $A_{t}(g)$ has limit points at asymptotic times $t$ which are closable operators having the dens of vectors $(1+\lambda H)^{-1-\varepsilon} \mathscr{H}$ in their respective domain. This a priori information greatly simplifies the analysis, notably in the case of massless particles [8].

In a somewhat different approach to collision theory which seems to be suitable for the discussion of particles as well as infraparticles, cf. [9] and references quoted there, Araki and Haag [5] have studied the timelike asymptotic behaviour of almost local observables which can be interpreted as detectors. A typical example of such an observable is $C=B^{*} B$, where $B$ is any almost local operator whose space-time Fourier transform has compact support in the complement of the closed forward lightcone. The operator $B$ thus annihilate vacuum state.

Araki and Haag proved that in theories of massive particles the spatial averages

$$
C(t ; h)=\left.\int d^{s} x h\left(\mathbf{x} / x_{0}\right) C\left(x_{0}, \mathbf{x}\right)\right|_{x_{0}=t}
$$

of the operators $C$, where $h$ is any essentially bounded function on $\mathbb{R}^{s}$, converge in the sense of bilinear forms at asymptotic times $t$ to the asymptotic momentum space densities of the respective particles. These operators can therefore be used 
to determine directly the collision cross sections without appeal to the scattering matrix.

As in the case of asymptotic fields the asymptotic behaviour of the operators $C(t ; h)$ can be controlled on cleverly chosen domains of collision states [5]. In the presence of interaction these domains are different, however, for large positive and negative times $t$ and therefore do not include all states of physical interest.

The present results show that the situation is actually much better than one might infer from the discussion in [5]. If one restricts attention to the sufficiently rich set of observables of the form $C=B^{*} B$, where $B$ has the properties stated above, it follows from Lemma 2.2 that for any $E>0$

$$
\|C(t ; h) P(E)\| \leq \text { const } \cdot\|h\|_{\infty}
$$

uniformly in $t$. For the proof of this assertion we decompose $B$ into a finite sum of almost local operators $B_{i}$ whose space-time Fourier transforms have support in compact and convex subsets of the complement of the closed forward lightcone. There then exists a number $n$ such that $B_{i}\left(\mathbf{x}_{1}\right) \ldots B_{i}\left(\mathbf{x}_{n}\right) P(E)=0$ for all operators $B_{i}$ and all $\mathbf{x}_{1}, \ldots, \mathbf{x}_{n} \in \mathbb{R}^{s}$. We therefore obtain from Lemma 2.2 for any compact subset $\mathbf{K} \subset \mathbb{R}^{s}$ the bound

$$
\left\|P(E) \int_{\mathbf{K}} d^{s} x\left(B_{i}^{*} B_{i}\right)(\mathbf{x}) P(E)\right\| \leq(n-1) \cdot \int d^{s} x\left\|\left[B_{i}(\mathbf{x}), B_{i}^{*}\right]\right\|,
$$

where the integral on the right-hand side exists since the operators $B_{i}$ are almost local. Since $B=\sum_{1}^{m} B_{i}$ and consequently $C=B^{*} B \leq 2^{m} \cdot \sum_{1}^{m} B_{i}^{*} B_{i}$, we conclude that for any $E \geq 0$,

$$
\left\|P(E) \int_{\mathbf{K}} d^{s} x h\left(\mathbf{x} / x_{0}\right) C\left(x_{0}, \mathbf{x}\right) P(E)\right\| \leq \mathrm{const} \cdot\|h\|_{\infty}
$$

uniformly in $\mathbf{K}$ and $x_{0}$. From this bound and the fact that $C$ is a positive operator we infer that the integral $\int d^{s} x h\left(\mathbf{x} / x_{0}\right) C\left(x_{0}, \mathbf{x}\right)$ exists in the sense of bilinear forms between vectors of finite energy. Moreover, since the space-time Fourier transform of $C(x)$ has compact support, the estimate (18) stays true if one replaces the projection $P(E)$ on the left-hand side of the integral by 1 . Hence the integral $\int d^{s} x h\left(\mathbf{x} / x_{0}\right) C\left(x_{0}, \mathbf{x}\right)$ is also defined as an operator on the dense set of vectors of finite energy, and the stated inequality (16) follows.

Similarly to the case of the asymptotic fields the bound (16) shows that the sequence of operators $C(t ; h)$ has limit points at asymptotic times $t$ which are closable operators having all vectors of finite energy in their respective domain. Hence the convergence proofs in [5] can be extended to this substantially larger set of vectors which is independent of the asymptotic direction of $t$.

We emphasize that the existence of limit points of the sequence $C(t ; h)$ does not depend on the assumption that the theory has a conventional particle interpretation. This fact lends support to the conjecture that the results of Araki and Haag can be extended to theories involving infraparticles. A preliminary discussion of these perspectives can be found in [9], a more detailed analysis will be given in [10]. 
This brief account of applications may suffice to illustrate the utility of our general results. We expect that our simple method of exploiting "positivity", expounded in the proofs of Lemma 2.1 and Lemma 2.2, respectively, will be of further use in the structural analysis of quantum field theory.

\section{Appendix}

We give here the proof of Lemma 2.2. As in the case of Lemma 2.1 the proof is achieved by induction in $n$, and as before the statement is trivial for $n=1$. For the step from $n-1$ to $n$ we choose any state $\omega$ on $\mathscr{B}(\mathscr{H})$ which is induced by some vector $\Phi$ in $P_{n} \mathscr{H}$ and set $Q=\int_{\mathbf{K}} d^{s} x\left(B^{*} B\right)(\mathbf{x})$. We have

$$
\begin{aligned}
\omega(Q \cdot Q)= & \int_{\mathbf{K}} d^{s} x \int_{\mathbf{K}} d^{s} y\left\{\omega\left(B^{*}\left(\mathbf{x}\left[B(\mathbf{x}), B^{*}(\mathbf{y})\right] B(\mathbf{y})\right)+\omega\left(B^{*}(\mathbf{x}) B^{*}(\mathbf{y}) B(\mathbf{x}) B(\mathbf{y})\right)\right\}\right. \\
\leq & \int_{\mathbf{K}} d^{s} x \int_{\mathbf{K}} d^{s} y\left\{\|B(\mathbf{x}) \Phi\|^{1 / 2}\|B(\mathbf{y}) \Phi\|^{1 / 2} \|\left[B(\mathbf{x}), B^{*}(\mathbf{y})\right]\right. \\
& \times B(\mathbf{y}) \Phi\left\|^{1 / 2}\right\|\left[B(\mathbf{y}), B^{*}(\mathbf{x})\right] B(\mathbf{x}) \Phi \|^{1 / 2} \\
& \left.+\omega\left(B^{*}(\mathbf{x})\left(B^{*} B\right)(\mathbf{y}) B(\mathbf{x})\right)^{1 / 2} \omega\left(B^{*}(\mathbf{y})\left(B^{*} B\right)(\mathbf{x}) B(\mathbf{y})\right)^{1 / 2}\right\} \\
\leq & \int_{\mathbf{K}} d^{s} x \int_{\mathbf{K}} d^{s} y\left\{\|B(\mathbf{y}) \Phi\|\left\|\left[B(\mathbf{x}), B^{*}(\mathbf{y})\right] B(\mathbf{y}) \Phi\right\|\right. \\
& \left.+\omega\left(B^{*}(\mathbf{x})\left(B^{*} B\right)(\mathbf{y}) B(\mathbf{x})\right)\right\} \\
\leq & \omega(Q) \cdot \sup _{\mathbf{y}} \int_{\Delta \mathbf{K}} d^{s} x \frac{\left\|\left[B(\mathbf{x}), B^{*}\right] B U(-\mathbf{y}) \Phi\right\|}{\|B U(-\mathbf{y}) \Phi\|}+\int_{\mathbf{K}} d^{s} x \omega\left(B^{*}(\mathbf{x}) Q B(\mathbf{x})\right),
\end{aligned}
$$

where we made use of the Cauchy-Schwarz inequality and the fact that the geometric mean of two numbers is smaller than their arithmetic mean. Since $\Phi \in P_{n} \mathscr{H}$ the vectors $B U(-\mathbf{y}) \Phi$ and $B(\mathbf{x}) \Phi$ are elements of $P_{n-1} \mathscr{H}$. We can therefore proceed from the preceding estimate to

$$
\omega(Q \cdot Q) \leq \omega(Q) \cdot\left\{\sup \int_{\Delta \mathbf{K}} d^{s} x\left\|\left[B(\mathbf{x}), B^{*}\right] \Psi\right\|+\left\|P_{n-1} Q P_{n-1}\right\|\right\}
$$

where the supremum is to be taken with respect to all unit vectors $\Psi \in P_{n-1} \mathscr{H}$. Making use of the induction hypothesis and the facts that $\omega(Q \cdot Q) \geq \omega(Q)^{2}$ and $P_{i} \leq P_{k}$ for $i \leq k$ we conclude that

$$
\omega(Q) \leq(n-1) \cdot \sup \int_{\Delta \mathbf{K}} d^{s} x\left\|\left[B(\mathbf{x}), B^{*}\right] \Psi\right\| .
$$

The statement now follows by taking the supremum over all states $\omega$ which are induced by vectors in $P_{n} \mathscr{H}$.

Acknowledgements. I gratefully acknowledge the hospitality of Prof. J. Lewis at the Dublin Institute for Advanced Studies where this paper was written. I am also grateful for financial support from this institution as well as for a travel grant from the DFG, Federal Republic of Germany. 


\section{References}

1. Haag, R., Schroer, B.: Postulates of quantum field theory. J. Math. Phys. 3, 248-256 (1962)

2. Arveson, B.: The harmonic analysis of automorphism groups. In: Operator algebras and applications. I. Proceedings of Symposia in Pure Mathematics, Vol. 38. Providence: Am. Math. Soc. 1982

3. Buchholz, D., Wanzenberg, R.: In preparation

4. Araki, H.: Einführung in die axiomatische Quantenfeldtheorie. II. Lecture notes. ETH Zürich 1962

5. Araki, H., Haag, R.: Collision cross sections in terms of local observables. Commun. Math. Phys. 4, 77-91 (1967)

6. Reed, M., Simon, B.: Methods of modern mathematical physics. II. Fourier analysis, self adjointness. New York, San Francisco, London: Academic Press 1975

7. Lehmann, H., Symanzik, K., Zimmermann, W.: Zur Formulierung quantisierter Feldtheorien. Nuovo Cimento 1, 205-225 (1955)

8. Buchholz, D.: Collision theory for massless Bosons. Commun. Math. Phys. 52, 147-173 (1977)

9. Buchholz, D.: On particles, infraparticles, and the problem of asymptotic completeness. In: VIIIth International Congress on Mathematical Physics, Marseille 1986. Singapore: World Scientific 1987

10. Buchholz, D., Stein, U.: In preparation

Communicated by H. Araki

Received September 30, 1989 
